\title{
Mixed infection zones may be important in the epidemiology of contagious agalactia
}

\author{
Amirreza Jafarizadeh ${ }^{1}$, Seyed Ali Pourbakhsh ${ }^{2}$, \\ Keyvan Tadayon ${ }^{3}$, Mahmud Jamshidian ${ }^{1}$, Abbas Ashtari ${ }^{2}$ \\ ${ }^{1}$ Department of Microbiology, Faculty of Veterinary Sciences, Science and Research Branch, \\ Islamic Azad University, 477893855, Tehran, Iran \\ ${ }^{2}$ Mycoplasma Reference Laboratory, ${ }^{3}$ Department of Aerobic Bacterial Vaccine Production, \\ Razi Vaccine and Serum Research Institute, 3197619751, Karaj, Iran \\ a.jafarizade@gmail.com
}

Received: November 26, 2015 Accepted: May 12, 2016

\begin{abstract}
Introduction: The current study was designed to detect Mycoplasma agalactiae (Ma), Mycoplasma mycoides subsp. capri (Mmc), Mycoplasma capricolum subsp. capricolum (Mcc) and Mycoplasma putrefaciens (Mp) in sheep and goats with clinical signs consistent with contagious agalactia. Material and Methods: A total of 299 samples were collected from 55 mixed herds in Azarbaijan-e-Sharghi province, Iran. Samples were examined using PCR and culture methods. Results: The findings showed that in 40 herds at least one sample was positive by PCR or culture method. Moreover, out of 274 sheep samples, 101 were proved to be positive using the PCR technique and 76 were found positive using the culture method. Out of 25 goat samples, 10 were found positive using PCR and 9 were positive through the culture method. Less than $20 \%$ of isolated mycoplasmas were $M a$. $M a$ was detected from almost all studied regions in the province while $M m c, M c c$, and $M p$ were detected only in a very limited area that was deemed by the research group the mixed infection zone. Conclusion: In vaccination or eradication projects, it would be more economical to focus on mixed infection zones. Further investigation on mixed infection zones could facilitate better understanding of contagious agalactia epidemiology.
\end{abstract}

Keywords: bovidae, contagious agalactia, Mycoplasma, bacteriological analysis, Iran.

\section{Introduction}

Contagious agalactia (CA), one of the most important diseases of sheep and goats in the Mediterranean and Middle Eastern countries, is characterised by mastitis, arthritis, keratoconjunctivitis, and occasionally abortion. The classic agent of CA is Mycoplasma agalactiae (Ma). However, Mycoplasma mycoides subsp. capri (Mmc), Mycoplasma capricolum subsp. capricolum (Mcc), and Mycoplasma putrefaciens $(\mathrm{Mp})$ usually cause a clinically similar disease, doing so more often in goats than in sheep (13). Based on epidemiological studies, there are some obstacles to controlling the disease, namely asymptomatic carriers and variable surface antigens, which make protection against CA more difficult in sheep and goats (7). According to the OIE report, although vaccination is routine in endemic countries, commercial vaccines are not considered to be very effective (13). In Iran, where there is a high population of small ruminants with large numbers being milked by traditional methods, many cases of CA are reported (12). Moreover, the high expense of CA treatment leads to frequent suppression of reports of such cases, especially when they are sporadic, which is common for Iran. The purpose of the present study is to detect the causative agents of $\mathrm{CA}$ in an Iranian province located close to the border of Azerbaijan and Armenia.

\section{Material and Methods}

Sampling. Sampling was carried out from June 2011 to October 2012. Samples to a total of 299 were collected from 55 mixed sheep and goat herds in Azarbaijan-e-Sharghi province. Samples were collected from milk, joint punctures, ear canals, and eyes of the animals with clinical signs or history of CA. During the 
sampling phase, $1 \mathrm{~mL}$ of milk was taken by hand into a sterile tube. The joint fluid sample was taken using a sterile syringe, and ear canal and eye conjunctiva samples were collected by sterile swabs. The samples were transferred by transport medium to the Mycoplasma Reference Laboratory of Razi Vaccine and Serum Research Institute, Karaj, Iran.

Mycoplasma cultures. PPLO broth and PPLO agar medium (BBL, Becton, Dickinson and Company, USA) were used for mycoplasma culture. Inoculated cultures were incubated at $37^{\circ} \mathrm{C}$ in a humidified atmosphere with $5 \% \mathrm{CO}_{2} . M a$ reference strain (NCTC 10123) was used as a positive control and uncultured PPLO broth as a negative control. Cultures were examined daily to detect any signs of growth. Typical mycoplasma colonies were observed under microscopic examination in positive PPLO agar cultures. PPLO broth cultures were determined positive by colour change and fine cloudiness in the medium. Glycerol was added to the positive PPLO broth cultures and they were then frozen at $-70^{\circ} \mathrm{C}$ for long-term storage $(11,12)$.

DNA extraction and PCR. DNA was extracted from samples by conventional procedures (10) and examined by PCR to identify genus-positive samples. All genus-positive samples were tested by a specific PCR procedure for M. mycoides cluster and $M a$. Finally, all $M$. mycoides cluster-positive samples were examined by a separate PCR procedure to detect $M m c$,
$M c c$, and $M p . M a$ reference strain (NCTC 10123), Mcc reference strain (NCTC 10154), Mmc reference strain (NCTC 11706), and $M p$ reference strain (NCTC 10155) were used as positive controls. Uncultured PPLO broth was used as a negative control. Table 1 shows the primers used for PCR.

The PCR was performed by conventional procedures as described by the manufacturer (CinnaGen Co., Iran). The PCR assay was conducted in a gradient Mastercycler (Eppendorf AG, Germany). Visualisation of amplified products was performed by UV illumination after electrophoresis (1\% agarose gel in $1 \times$ Tris-acetic acid-EDTA (TAE) buffer) and ethidium bromide staining $(14,15)$.

\section{Results}

Among all 274 sheep samples, 36.8\% were positive for the mycoplasma genus. About $14 \%$ of mycoplasmas were $M a$. Ma was detected from almost all regions in the province while $M m c, M c c$, and $M p$ were detected within a very limited zone that the research group termed the mixed infection zone (Fig. 1). Table 2 shows the results in detail.

Among 25 goat samples, nearly $40 \%$ were positive for the mycoplasma genus by both culture and PCR methods. (Fig. 2). Table 3 shows the results in detail.

Table 1. Nucleotide sequences of the primers used for PCR

\begin{tabular}{|c|c|c|}
\hline Species & Primers & References \\
\hline M. agalactiae & $\begin{array}{l}\text { FS1-5'-AAAGGTGCTTGAGAAATGGC-3' } \\
\text { FS2-5'-GTTGCAGAAGAAAGTCCAATCA-3' }\end{array}$ & 18 \\
\hline M. mycoides cluster & $\begin{array}{l}\text { P1F-5'-TATATGGACTAAAAAGAC-3' } \\
\text { P2R-5'-AATGCATCATAAATAATTG-3' }\end{array}$ & 8 \\
\hline M. mycoides subsp. capri & $\begin{array}{l}\text { P4F-5'-ACTGAGCAATTCCTCTT-3' } \\
\text { P5R-5'-TTAAATAAGTTTGTATATGAAT-3' }\end{array}$ & 8 \\
\hline M. capricolum subsp. capricolum & $\begin{array}{l}\text { P4F-5'-ACTGAGCAATTCCTCTT-3' } \\
\text { P8R-5'-GTAAACCGTGTATATCAAAT-3' }\end{array}$ & 8 \\
\hline M. putrefaciens & $\begin{array}{l}\text { SSF1-5'-GCGGCATGCCTAATACATGC-3' } \\
\text { SSR1-5'-AGCTGCGGCGCTGAGTTCA-3' }\end{array}$ & 12 \\
\hline
\end{tabular}

Table 2. Results of sheep sample testing

\begin{tabular}{llllllll}
\hline Sample type & Number of samples & Culture $^{*}$ & PCR Genus $^{*}$ & ${\text { PCR } M a^{*}}^{*}$ & PCR $M m c^{*}$ & PCR $M c c^{*}$ & ${\text { PCR } M p^{*}}^{*}$ \\
\hline Milk & 74 & 16 & 26 & 4 & 1 & 1 & 0 \\
Ear canal swab & 126 & 35 & 43 & 6 & 0 & 1 & 1 \\
Eye swab & 72 & 25 & 32 & 4 & 2 & 2 & 1 \\
Joint puncture & 2 & 0 & 0 & 0 & 0 & 0 & 0 \\
\hline Total & 274 & 76 & 101 & 14 & 3 & 4 & 2 \\
\hline
\end{tabular}

* Number of positive results

Table 3. Results of goat sample testing

\begin{tabular}{|c|c|c|c|c|c|c|c|}
\hline Sample Type & Number of samples & Culture $^{*}$ & PCR Genus* & PCR $M a^{*}$ & PCR $M m c^{*}$ & PCR $M c c^{*}$ & PCR $M p^{*}$ \\
\hline Milk & 13 & 6 & 6 & 2 & 0 & 0 & 0 \\
\hline Ear canal swab & 9 & 2 & 3 & 0 & 0 & 0 & 0 \\
\hline Eye swab & 3 & 1 & 1 & 0 & 0 & 0 & 0 \\
\hline Joint puncture & 0 & 0 & 0 & 0 & 0 & 0 & 0 \\
\hline Total & 25 & 9 & 10 & 2 & 0 & 0 & 0 \\
\hline
\end{tabular}

* Number of positive results 


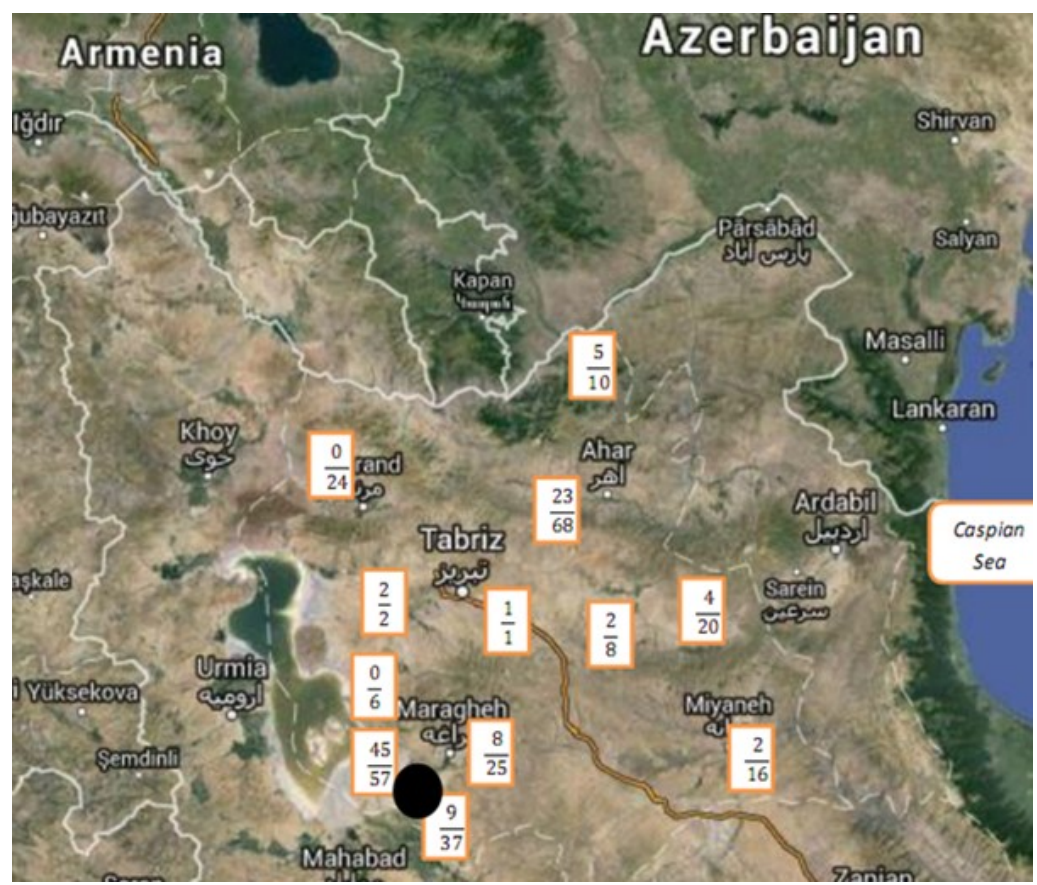

Fig. 1. Geographic distribution of sheep sampling regions. Denominator digits show the number of samples. Numerator digits show the number of genus-positive samples by PCR. $M m c, M c c$, and $M p$ were detected only in the mixed infection zone (black zone)

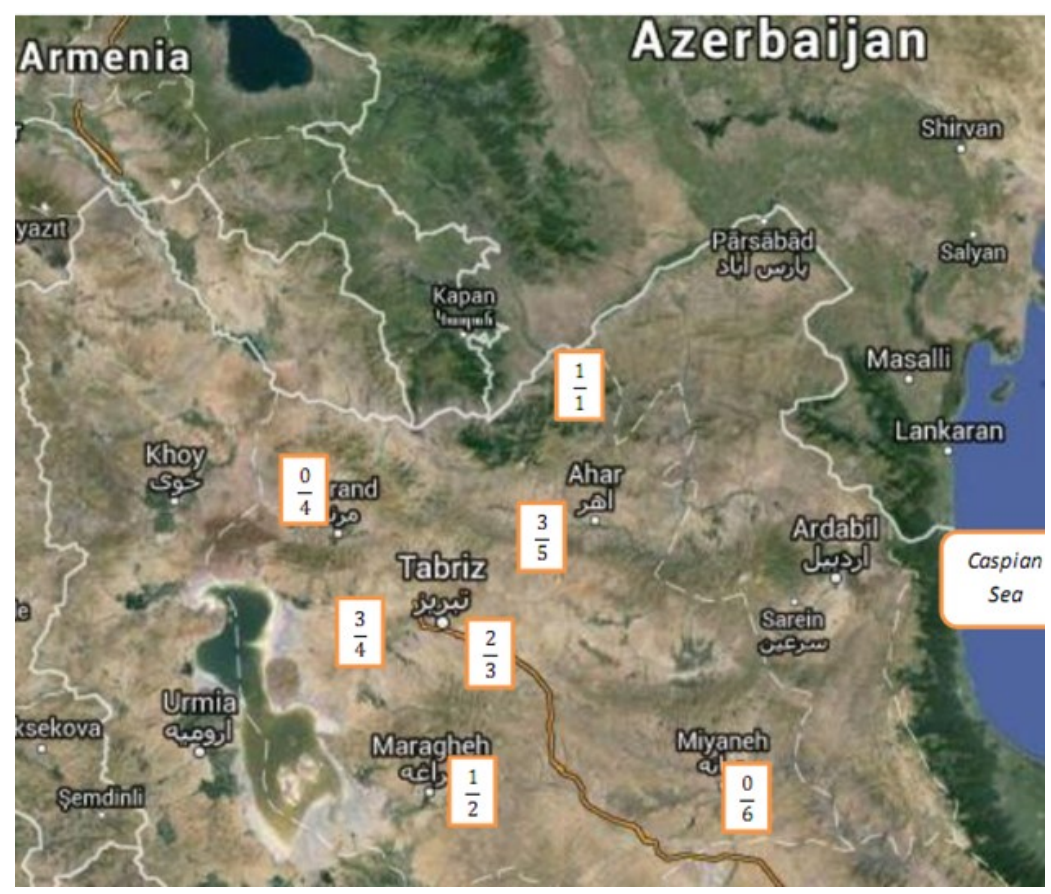

Fig. 2. Geographic distribution of sheep sampling regions. Denominator digits show the number of samples. Numerator digits show the number of genus-positive samples by PCR

\section{Discussion}

In this study, $73 \%$ of the mixed herds showed at least one positive PCR or culture result, which is in line with available literature data revealing that Iran has always had a problem with CA (13). Among the 12 sampled regions, only two of them were free of mycoplasmas. Mycoplasma-free regions were sampled only once, so more sampling may change the results, because it is known that the excretion of mycoplasmas in milk and semen is periodic $(3,6)$.

There was a difference between results obtained by PCR and culture methods. Antibiotic therapy before sampling and the time interval between sampling and transportation may have reduced the number of viable mycoplasmas. Generally, PCR shows high sensitivity 
in mycoplasma detection (11). In agreement with this study, PCR has also led to more positive results in other provinces of Iran $(9,14,15)$.

Surprisingly, it was found in the study that more than $80 \%$ of isolated mycoplasmas were not $M a, M c c$, $M m c$, or $M p$, which requires further investigations. Typical mycoplasma colonies were observed in $75 \%$ of the positive samples, which assures us of the genus of the isolated microorganisms. Moreover, in almost all published papers in Iran $M a$ is not the major agent of CA $(1,9,14,17)$. In Jordan, another Middle Eastern country, molecular studies indicated that less than 25\% of mycoplasma isolates from small ruminants are Ma (2). Moreover, in France, M. arginini was the major species isolated from sheep flocks (4). It seems, there is need to study other species of mycoplasmas in the future.

In Iran, as in other countries in the Middle East and Africa, mixed herd farming of sheep, goats, and even cows is common. This type of shepherding provides opportunities for recombination and horizontal gene transfer between different species of ruminant mycoplasmas. About $30 \%$ of the exchanged genes have a major role in mycoplasma interaction with host cells and as a result in the virulence $(5,16)$. In this study, $M m c, M c c$, and $M p$ were detected within a very limited area that was designated the mixed infection zone. The zone is distinguished by unsatisfactory vaccination results, so it could be also suggested that a relationship may exist between the mixed infection zone and CA vaccination failure. Strict rules on livestock selling as well as vaccination against certain species of mycoplasmas in the mixed infection zone might be effective in CA control. Finally, future studies should address the important research issue regarding the possibility of finding other patterns similar to the mixed infection zone in other provinces of Iran and also in other countries.

Conflict of Interests Statement: The authors declare that they have no conflict of interests regarding the publication of this article.

Financial Disclosure Statement: This study was supported by the Education and Research Deputy of Jihad-e- Keshavarzi Ministry with the grant No. 2-1818-89067.

Animal Rights Statement: The authors declare that the experiments on animals were conducted in accordance with the local Ethical Committee's guidelines.

Acknowledgements: We are grateful to Robin A.J. Nicholas for his friendly help with the writing of the manuscript. We also thank all staff of the Mycoplasma Reference Laboratory, Razi Vaccine and Serum Research Institute, Karaj, Iran.

\section{References}

1. Abtin A.R., Pourbakhsh S.A., Ashtari A., Bayatzadeh M.A., Barani S.M., Ahangaran S.: Isolation and identification of Mycoplasma agalactiae by culture and polymerase chain reaction (PCR) from sheep of Qom province, Iran. Arch Razi Inst 2013, 68, 11-16.

2. Al-Momani W., Halablab M.A., Abo-Shehada M.N., Miles K., McAuliffe L., Nicholas R.A.J.: Isolation and molecular identification of small ruminant mycoplasmas in Jordan. Small Rum Res 2006, 65, 106-112.

3. Ariza-Miguel J., Rodriguez-Lazaro D., Hernandez M.: A survey of Mycoplasma agalactiae in dairy sheep farms in Spain. BMC Vet Res 2012, 8, 171-176.

4. Chazel M., Tardy F., Le Grand D., Calavas D., Poumarat F.: Mycoplasmoses of ruminants in France: recent data from the national surveillance network. BMC Vet Res 2010, 6, 32.

5. Chopra-Dewasthaly R., Baumgartner M., Gamper E., Innerebner C., Zimmermann M., Schilcher F., Tichy A., Winter P., Jechlinger W., Rosengarten R., Spergser J.: Role of Vpma phase variation in Mycoplasma agalactiae pathogenesis. FEMS Immunol Med Microbiol 2012, 66, 307-22.

6. De la Fe C., Amores J., Gomez Martın A., Sanchez A., Contreras A., Corrales J.C.: Mycoplasma agalactiae detected in the semen of goat bucks. Theriogenology 2009, 72, 1278-1281.

7. Gomez-Martin A., Amores J., Paterna A., De la Fe C.: Contagious agalactia due to Mycoplasma spp. in small dairy ruminants: epidemiology and prospects for diagnosis and control. Vet J 2013, 198, 48-56.

8. Hotzel H., Sachse K., Pfutzner H.: A PCR scheme for differentiation of organisms belonging to the Mycoplasma mycoides cluster. Vet Microbiol 1996, 49, 31-43.

9. Khezri M., Pourbakhsh S.A., Ashtari A., Rokhzad B.: Investigation of Mycoplasma agalactiae in sheep in Kurdistan province by PCR. Arch Razi Inst 2015, 70, 73-80.

10. Kojima A., Takahashi T., Kijima M., Ogikubo Y., Nishimura M., Nishimura S., Harasawa R., Tamura Y.: Detection of Mycoplasma in avian live virus vaccines by polymerase chain reaction. Biologicals 1997, 25, 365-371.

11. May M., Balish M.F., Blanchard A.: The Order Mycoplasmatales. In: The Prokaryotes by Rosenberg E., DeLong E.F., Lory S., Stackebrandt, E., Thompson, F., Springer-Verlag, Berlin Heidelberg, 2014, 515-550.

12. Nicholas R., Ayling R., McAuliffe L.: Mycoplasma Diseases of Ruminants, CABI, Wallingford, UK, 2008.

13. OIE. Manual of Diagnostic Tests and Vaccines for Terrestrial Animals, Paris, 2015. Chapter 2.7.5. Contagious Agalactia.

14. Pooladgar A.R., Looni R., Ghaemmaghami S., Pourbakhsh A., Ashtari A., Ali-Shirudi A.: Isolation and identification of Mycoplasma agalactiae by culture and polymerase chain reaction (PCR) from affected sheep to contagious agalactia of Khuzestan province, Iran. Arch Razi Inst 2015, 70, 21-27.

15. Pourbakhsh S.A., Abtin A.R., Ashtari A., Bayatzadeh M.A., Barani S.M., Asli E.: Detection of Mycoplasma capricolum capricolum from goats of Qom province, Iran. Arch Razi Inst 2015, 70, 45-50.

16. Sirand-Pugnet P., Lartigue C., Marenda M., Jacob D., Barre A., Barbe V., Schenowitz C., Mangenot S., Couloux A., Segurens B., De Daruvar A., Blanchard A., Citti C.: 2007. Being pathogenic, plastic, and sexual while living with a nearly minimal bacterial genome. PLoS Genet 2007, 3, e75.

17. Sotoodehnia A., Aarabi I.: Agalactia disease and its geographical distribution in sheep and goats in Iran. Arch Razi Inst 1986, $36,75-78$.

18. Tola S., Idini G., Manunta D., Galleri G., Angioi A., Rocchigiani A.M., Leori G.: Rapid and specific detection of Mycoplasma agalactiae by PCR. Vet Microbiol 1996, 51, 77-84. 